\title{
Despite worse baseline status depressed patients achieved outcomes similar to those in nondepressed patients after surgery for cervical deformity
}

\author{
Gregory W. Poorman, BA, ${ }^{1}$ Peter G. Passias, MD, ${ }^{1}$ Samantha R. Horn, BA, ${ }^{1}$ \\ Nicholas J. Frangella, BS, ${ }^{1}$ Alan H. Daniels, MD, ${ }^{2}$ D. Kojo Hamilton, MD, ${ }^{3}$ Hanjo Kim, MD, ${ }^{4}$ \\ Daniel Sciubba, MD, ${ }^{5}$ Bassel G. Diebo, MD, ${ }^{6}$ Cole A. Bortz, BA, ${ }^{1}$ Frank A. Segreto, BS, ${ }^{1}$ \\ Michael P. Kelly, MD, ${ }^{7}$ Justin S. Smith, MD, PhD, ${ }^{8}$ Brian J. Neuman, MD, ${ }^{5}$ \\ Christopher I. Shaffrey, MD, ${ }^{8}$ Virginie LaFage, MD, ${ }^{4}$ Renaud LaFage, MS, ${ }^{4}$ \\ Christopher P. Ames, MD, ${ }^{9}$ Robert Hart, MD, ${ }^{10}$ Gregory M. Mundis Jr., MD, ${ }^{11}$ and \\ Robert Eastlack, MD, ${ }^{11}$ on behalf of the International Spine Study Group
}

1Department of Orthopaedic Surgery, NYU Hospital for Joint Diseases, New York, New York; ${ }^{2}$ Department of Orthopaedic Surgery, Brown University Alpert Medical School, Providence, Rhode Island; '3Department of Neurologic Surgery, University of Pittsburgh, Pennsylvania; ${ }^{4}$ Department of Orthopaedic Surgery, Hospital for Special Surgery, New York, New York; ${ }^{5}$ Department of Neurologic Surgery, Johns Hopkins Medical Center, Baltimore, Maryland; ${ }^{6}$ Department of Orthopaedic Surgery, University Hospital of Brooklyn, New York, New York; 'Department of Orthopaedic Surgery, Washington University, St. Louis, Missouri; ${ }^{8}$ Department of Neurological Surgery, University of Virginia Health System, Charlottesville, Virginia; ${ }^{9}$ Department of Neurological Surgery, University of California, San Francisco, California; ${ }^{10}$ Swedish Neuroscience Institute, Seattle, Washington; and ${ }^{11}$ San Diego Center for Spinal Disorders, La Jolla, California

OBJECTIVE Depression and anxiety have been demonstrated to have negative impacts on outcomes after spine surgery. In patients with cervical deformity (CD), the psychological and physiological burdens of the disease may overlap without clear boundaries. While surgery has a proven record of bringing about significant pain relief and decreased disability, the impact of depression and anxiety on recovery from cervical deformity corrective surgery has not been previously reported on in the literature. The purpose of the present study was to determine the effect of depression and anxiety on patients' recovery from and improvement after CD surgery.

METHODS The authors conducted a retrospective review of a prospective, multicenter CD database. Patients with a history of clinical depression, in addition to those with current self-reported anxiety or depression, were defined as depressed (D group). The D group was compared with nondepressed patients (ND group) with a similar baseline deformity determined by propensity score matching of the cervical sagittal vertical axis (cSVA). Baseline demographic, comorbidity, clinical, and radiographic data were compared among patients using t-tests. Improvement of symptoms was recorded at 3 months, 6 months, and 1 year postoperatively. All health-related quality of life (HRQOL) scores collected at these follow-up time points were compared using t-tests.

RESULTS Sixty-six patients were matched for baseline radiographic parameters: 33 with a history of depression and/ or current depression, and 33 without. Depressed patients had similar age, sex, race, and radiographic alignment: cSVA, T-1 slope minus C2-7 lordosis, SVA, and T-1 pelvic angle $(p>0.05)$. Compared with nondepressed individuals, depressed patients had a higher incidence of osteoporosis $(21.2 \%$ vs $3.2 \%, p=0.028)$, rheumatoid arthritis $(18.2 \%$ vs $3.2 \%, p=0.012$ ), and connective tissue disorders (18.2\% vs $3.2 \%, p=0.012)$. At baseline, the $\mathrm{D}$ group had greater neck pain (7.9 of 10 vs 6.6 on a Numeric Rating Scale [NRS], $p=0.015)$, lower mean EQ-5D scores (68.9 vs 74.7, $p<0.001$ ), but similar Neck Disability Index (NDI) scores ( 57.5 vs 49.9, $p=0.063$ ) and myelopathy scores (13.4 vs 13.9, $p=0.546)$.

ABBREVIATIONS $\mathrm{BMI}=$ body mass index; $\mathrm{CD}=$ cervical deformity; $\mathrm{CSVA}=$ cervical SVA; HRQOL = health-related QOL; ISSG = International Spine Study Group; $\mathrm{mJOA}=$ modified Japanese Orthopaedic Association; NDI = Neck Disability Index; NRS = Numeric Rating Scale; QOL = quality of life; SVA = sagittal vertical axis; TS-CL = T-1 slope minus C2-7 lordosis. 
Surgeries performed in either group were similar in terms of number of levels fused, osteotomies performed, and correction achieved (baseline to 3-month measurements) $(p<0.05)$. At 3 months, EQ-5D scores remained lower in the D group (74.0 vs 78.2, $p=0.044$ ), and NDI scores were similar (48.5 vs 39.0, $p=0.053$ ). However, neck pain improved in the D group (NRS score of 5.0 vs 4.3, $p=0.331$ ), and modified Japanese Orthopaedic Association (mJOA) scores remained similar (14.2 vs 15.0, $p=0.211$ ). At 6 months and 1 year, all HRQOL scores were similar between the 2 cohorts. Oneyear measurements were as follows: NDI 39.7 vs 40.7 ( $p=0.878)$, NRS neck pain score of 4.1 vs 5.0 ( $p=0.326)$, EQ-5D score of 77.1 vs $78.2(p=0.646)$, and mJOA score of 14.0 vs $14.2(p=0.835)$. Anxiety/depression levels reported on the EQ-5D scale were significantly higher in the depressed cohort at baseline, 3 months, and 6 months (all $p<0.05$ ), but were similar between groups at 1 year postoperatively (1.72 vs $1.53, p=0.416)$.

CONCLUSIONS Clinical depression was observed in many of the study patients with CD. After matching for baseline deformity, depression symptomology resulted in worse baseline EQ-5D and pain scores. Despite these baseline differences, both cohorts achieved similar results in all HRQOL assessments 6 months and 1 year postoperatively, demonstrating no clinical impact of depression on recovery up until 1 year after CD surgery. Thus, a history of depression does not appear to have an impact on recovery from CD surgery.

https://thejns.org/doi/abs/10.3171/2017.8.FOCUS17486

KEY WORDS cervical deformity; surgery; outcomes; spinal deformity

$\mathrm{C}$ ERVICAL deformity is a disabling condition resulting in not only impaired motor and physical functioning but also changes in appearance and mental well-being. The incidence of affective disorders such as depression and anxiety is high in patients with spinal disorders, including deformity. Determining the etiology of depression, whether in reaction to disability from deformity or as a stand-alone disease, is an important question in these patients with a high rate of comorbidities.

Depression and anxiety are well characterized in the literature as having a strong negative impact on postsurgery pain and outcome. ${ }^{14,18}$ Specifically, patients with anxiety and depression have reported higher levels of postsurgery pain and worse long-term outcomes. ${ }^{11,13}$

Currently there is no protocol for helping patients with depression and anxiety as they undergo cervical spine deformity surgery, whereas attempts at establishing protocols in the lumbar spine surgery population have been better developed. ${ }^{31}$ Similar recommendations for the cervical spine were summarized in a review published in the Journal of Orthopedic and Sports Physical Therapy. ${ }^{12}$ However, the authors of those recommendations only reported on 2 Level II trials and thus failed to provide definitive recommendations advising caregivers on how to cope with psychologically impaired patients. ${ }^{12}$ Furthermore, it is unclear whether the results of surgery alone adequately resolve the psychological and lifestyle variables strongly influencing neck pain and disability..$^{21,22,28,29}$

Given the interaction between the symptoms of radiculopathy, specifically neck and arm pain, and psychological distress, the consideration of various psychological factors and their associations with surgical outcome is crucial for joint decision making between surgeon and patient. As it currently stands, there is insufficient rationale for surgeons to use screening tools in identifying high-risk patients prior to surgery. With this in mind, we compared the pain experience and recovery of individuals with and without a history of depression as they underwent cervical deformity surgery. We used health-related quality of life (HRQOL) assessments in the form of the Neck Disability
Index (NDI), EuroQol's EQ-5D test, the modified Japanese Orthopaedic Association (mJOA) scale, and a quantitative assessment of back and neck pain (Numeric Rating Scale [NRS]) to compare surgical outcomes between a depressed (D group) and nondepressed (ND group) cohort.

We hypothesized that self-reported depression would be associated with worse patient-reported outcomes after cervical deformity surgery. This work, along with the established association of poor surgical outcome in patients with psychological distress, will emphasize the necessity for preoperative psychological care in populations with cervical deformity and depression so that optimal recovery and pain relief can be achieved.

\section{Methods}

Consecutive adult patients with cervical deformity were prospectively enrolled across 13 participating sites through the International Spine Study Group (ISSG). Institutional review board approval was obtained at each site.

Inclusion criteria for the study database were as follows: age $>18$ years, radiographically defined cervical deformity, and history of surgery to correct the deformity. Cervical deformity was defined as any one of the following markers: cervical kyphosis $\left(\mathrm{C} 2-7\right.$ sagittal Cobb angle $\geq 10^{\circ}$ ), cervical scoliosis $\left(\mathrm{C} 2-7\right.$ coronal Cobb angle $\left.\geq 10^{\circ}\right), \mathrm{C} 2-7$ sagittal vertical axis (SVA; C2-7) $\geq 4 \mathrm{~cm}$, or chin-brow vertical angle $\geq 25^{\circ}$ (Fig. 1). Patients with an active spinal neoplasm, spinal infection, or pregnancy were excluded.

Patient demographics, radiographic assessments, surgical/clinical details, complications, and patient-reported outcome scores were collected by the enrolling surgeon at baseline and at 3 months, 6 months, and 1 year after surgery.

Patients with depression were defined as those with a clinical diagnosis of depression (in patient history, not necessarily active) who also marked themselves as "....moderately anxious or depressed" or "...extremely anxious or depressed" on the EQ-5D questionnaire (one other option: "I am not anxious or depressed"). 

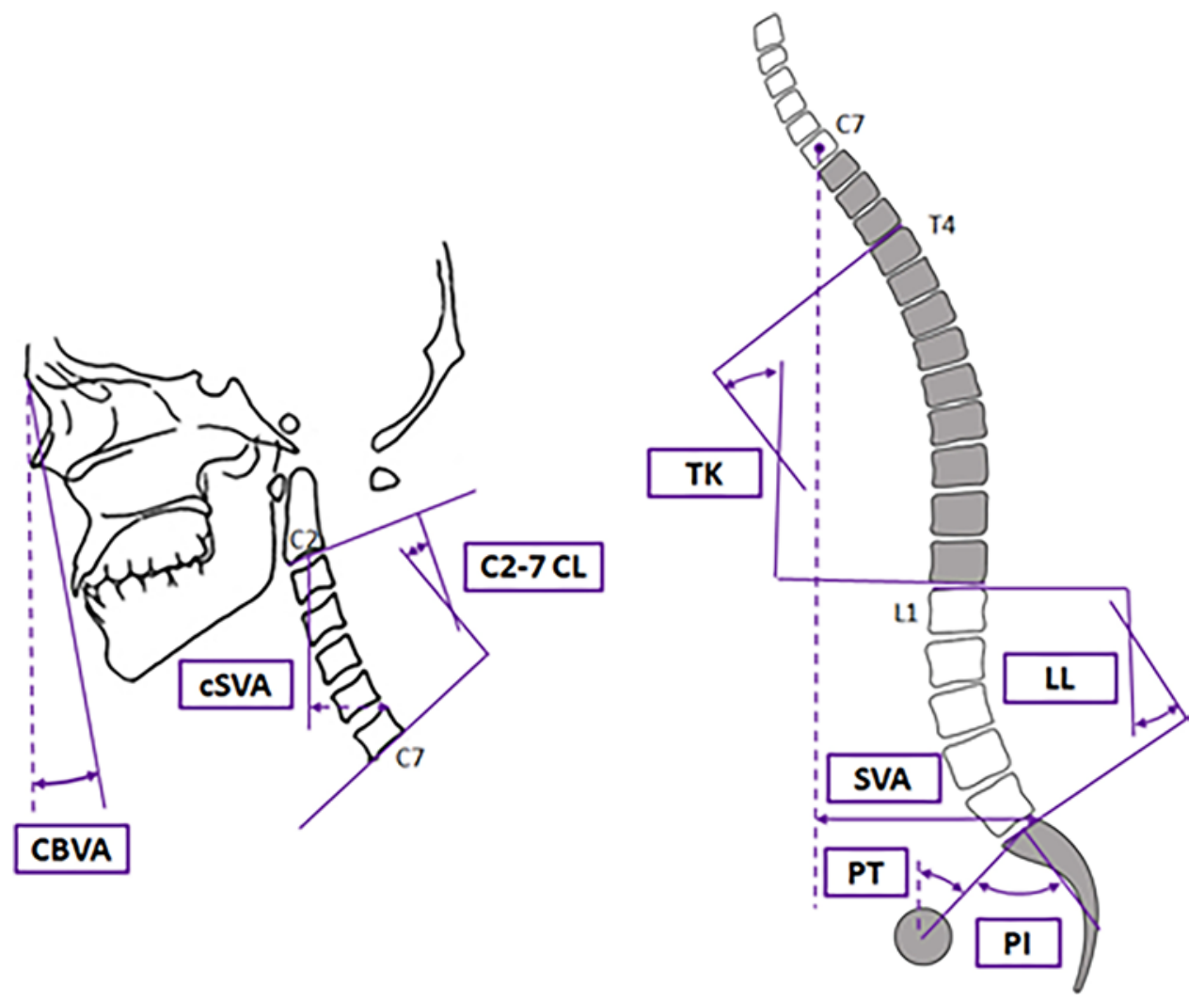

FIG. 1. Schematic of the sagittal alignment parameters measured for the cervical (left) and global spinopelvic (right) spinal regions. $\mathrm{C} 2-7 \mathrm{CL}$ = cervical lordosis; $\mathrm{CBVA}=$ chin-brow vertical angle; $\mathrm{LL}=$ lumbar lordosis; $\mathrm{PI}=$ pelvic incidence; $\mathrm{PT}=$ pelvic tilt; $\mathrm{TK}=$ thoracic kyphosis.

Primary analysis established differences in outcome score improvements between patients with and without baseline depression after they underwent surgical correction of cervical deformity.

\section{Primary Analysis}

Patients defined as currently depressed/anxious or with a history of depression/anxiety were propensity score matched to nondepressed/anxious patients according to cervical SVA (cSVA; offset from the C-2 plumb line and the posterosuperior corner of C-7) to control for differences in baseline deformity. Depressed versus nondepressed patient HRQOL scores were compared at baseline, 3 months, 6 months, and 1 year after surgery using t-tests. HRQOL scores queried included NDI, EQ-5D, mJOA, and NRS (back and neck pain) scores.

\section{Secondary Analysis}

A secondary descriptive analysis compared the 2 groups after matching demographics, surgical/clinical details, and radiographic changes. For continuous variables, normality was tested using a Kolmogorov-Smirnov test and compared using Student t-tests for normally distributed variables and Wilcoxon test for nonnormal data. Categorical variables were assessed using the chi-square or Fisher's exact test. Demographic variables included the following: sex, age, body mass index (BMI), Charlson Comorbidity Index scores, smoking status, history of cervical surgery, and diagnosis (congenital kyphosis, degenerative cervical kyphoscoliosis, degenerative cervical scoliosis, degenerative kyphosis, iatrogenic cervical kyphoscoliosis, iatrogenic kyphosis, traumatic kyphosis, other, or missing). Surgical/clinical details assessed were mean number of anterior levels fused, mean number of posterior levels fused, approach, osteotomy usage, and operative time. Finally, radiographic measurements included the cSVA, the T-1 slope minus C2-7 lordosis (TS-CL; mismatch between T-1 slope and cervical lordosis), lumbar lordosis (Cobb angle between L-1 inferior endplate and S-1 inferior endplate), thoracic kyphosis (Cobb angle between the T-4 inferior endplate and the T-12 inferior endplate), SVA (C-7 plumb line relative to the posterosuperior corner of S-1), pelvic incidence minus lumbar lordosis (mismatch between pelvic incidence and lumbar curvature), and pelvic tilt (angle between the vertical and the line through the sacral midpoint to the femoral head axis).

All statistical tests were 2-tailed. A p value $<0.05$ was considered statistically significant. All statistical analyses were performed using IBM SPSS Statistics, Version 23.0.

\section{Results \\ Patient Cohort}

A cohort of 33 patients with self-reported current and/or past clinical depression/anxiety (D group) were matched to 33 nondepressed patients (ND group). Demographically, patients were $65.5 \%$ female with a mean age of 60.0 years, 
TABLE 1. Summary of demographics in patients with and without a history of depression

\begin{tabular}{|c|c|c|c|}
\hline \multirow[b]{2}{*}{ Characteristic } & \multicolumn{2}{|c|}{$\begin{array}{l}\text { History of } \\
\text { Depression }\end{array}$} & \multirow[b]{2}{*}{$\begin{array}{c}p \\
\text { Value }\end{array}$} \\
\hline & $\begin{array}{c}\text { Yes } \\
(n=33)\end{array}$ & $\begin{array}{c}\text { No } \\
(n=33)\end{array}$ & \\
\hline Female (\%) & 69.7 & 61.3 & 0.487 \\
\hline Mean age (yrs) & 58.3 & 59.6 & 0.630 \\
\hline Mean BMI $\left(\mathrm{kg} / \mathrm{m}^{2}\right)$ & 30.4 & 30.0 & 0.827 \\
\hline Mean Charlson Comorbidity Index score & 0.73 & 0.61 & 0.659 \\
\hline Smoker (\%) & 3.2 & 9.5 & 0.097 \\
\hline Previous cervical surgery (\%) & 51.5 & 46.7 & 0.706 \\
\hline Diagnosis (no. of patients) & & & 0.849 \\
\hline Congenital kyphosis & 0 & 0 & \\
\hline Degenerative cervical kyphoscoliosis & 2 & 2 & \\
\hline Degenerative cervical scoliosis & 1 & 1 & \\
\hline Degenerative kyphosis & 16 & 16 & \\
\hline latrogenic cervical kyphoscoliosis & 1 & & \\
\hline latrogenic kyphosis & 4 & 6 & \\
\hline Traumatic kyphosis & 1 & 0 & \\
\hline Other & 7 & 5 & \\
\hline Missing & 1 & 3 & \\
\hline
\end{tabular}

BMI of 30.2, and a mean Charlson Comorbidity Index score of 0.75 (Table 1). All patients received long cervical fusions to correct cervical deformity (mean number of levels fused: 7.1; osteotomy frequency: $49.5 \%$; approach: $46.5 \%$ posterior only, $17.8 \%$ anterior only, and $35.7 \%$ combined; mean number of upper instrumented vertebrae: 2.8 ; and mean number of lowest instrumented vertebrae: 9.1) (Table 2).

\section{Radiographic Assessment}

Patients presented with deformities meeting the thresh-

TABLE 2. Summary of surgeries performed in patients with and without a history of depression

\begin{tabular}{lccc}
\hline \multicolumn{1}{c}{ Procedure } & \multicolumn{2}{c}{ History of Depression } & $p$ \\
\cline { 2 - 3 } & Yes $(\mathrm{n}=33)$ & No $(\mathrm{n}=33)$ & Value \\
\hline Mean no. of anterior levels fused & 1.5 & 1.8 & 0.616 \\
\hline Mean no. of posterior levels fused & 6.5 & 7.2 & 0.579 \\
\hline Approach (no. [\%]) & \multicolumn{3}{c}{} \\
\hline$\quad$ Anterior & $8(24.2)$ & $2(6.1)$ & 0.039 \\
\hline$\quad$ Posterior & $17(51.5)$ & $16(48.5)$ & 0.806 \\
\hline$\quad$ Combined & $8(24.2)$ & $14(42.4)$ & 0.117 \\
\hline $\begin{array}{l}\text { Three-column osteotomy (no. } \\
\text { [\%]) }\end{array}$ & $13(39.4)$ & $10(30.3)$ & 0.438 \\
\hline Operative time (mins) & & & \\
\hline Estimated blood loss (ml) & 645 & 398 & 0.614 \\
\hline
\end{tabular}

Boldface type indicates statistical significance (set at $p<0.05$ ).
TABLE 3. Summary of baseline radiographic alignment parameters in patients with and without a history of depression

\begin{tabular}{lccc}
\hline \multirow{2}{*}{ Baseline Parameter } & \multicolumn{2}{c}{ History of Depression } & $p$ \\
\cline { 2 - 3 } & Yes $(\mathrm{n}=33)$ & No $(\mathrm{n}=33)$ & Value \\
\hline cSVA $(\mathrm{mm})$ & 43.2 & 43.5 & 0.954 \\
\hline Cervical lordosis $(\mathrm{C} 2-7)\left({ }^{\circ}\right)$ & -9 & -4.4 & 0.362 \\
\hline T-1 slope minus cervical lordosis $\left(^{\circ}\right)$ & 35.3 & 34.0 & 0.764 \\
\hline Lumbar lordosis $\left({ }^{\circ}\right)$ & 55.5 & 49.0 & 0.159 \\
\hline Thoracic kyphosis $\left(^{\circ}\right)$ & 35.9 & 36.8 & 0.826 \\
\hline C7-S1 SVA (mm) & -2.3 & 1.2 & 0.849 \\
\hline $\begin{array}{l}\text { Pelvic incidence minus lumbar } \\
\text { lordosis }\left({ }^{\circ}\right)\end{array}$ & -0.8 & 3.1 & 0.442 \\
\hline Pelvic tilt $\left({ }^{\circ}\right)$ & 17.9 & & \\
\hline
\end{tabular}

olds established in the Ames cervical deformity classification system. ${ }^{3}$ In terms of cSVA, $63 \%$ of patients met the deformity cutoff at $>4 \mathrm{~cm}$, with $8 \%$ meeting the severe deformity cutoff at $>8 \mathrm{~cm}$. In terms of the TS-CL value, $92.7 \%$ of patients were symptomatic at $>15^{\circ}$, and $87 \%$ met severe cutoff for TS-CL deformity at $>20^{\circ}$. In terms of myelopathy, $7.8 \%$ of patients did not have myelopathy symptoms and $32.8 \%$ had mild, $39.7 \%$ moderate, and $19.8 \%$ severe symptoms. All other baseline radiographic parameters between D group and ND group patients are presented in Table 3.

In analyzing patient realignment procedures, patients matched for baseline cSVA showed similar postoperative alignment. Matched patients had statistically similar measurements for cervical lordosis (C2-7) and T1 slope (angle $\mathrm{C} 2-7)$ as well. This trend extended to all measurements of deformity for matched patients.

\section{HRQOL Assessment}

After graphically analyzing the results for the NDI scores, no statistical difference was found between the D and ND cohorts at any point during data collection (Fig. 2). At all time points, 2-tailed t-test analysis yielded $\mathrm{p}>$ 0.05 .

Depressed patients presented with significantly lower overall EQ-5D scores at baseline (0.69 [D group] vs 0.75 [ND group], $\mathrm{p}<0.001)$ and 3 months postsurgery (0.74 [D group] vs 0.78 [ND group], $p=0.044$, Fig. 3). Statistical difference diminished at the 6-month and 1-year marks (p $>0.05)$. EQ-5D anxiety/depression question scores were significantly higher in depressed patients at baseline (2.24 vs $1.67, \mathrm{p}<0.001), 3$ months (1.77 vs $1.38, \mathrm{p}=0.026)$, and 6 months $(1.90$ vs $1.33, p=0.004)$, but were similar at 1 year (1.72 vs $1.53, \mathrm{p}=0.416)$.

mJOA scores were found to be similar between groups at all points of data collection ( $p>0.05$, Fig. 4).

Measurements of the neck pain in these individuals showed the D group to have a significantly higher baseline average neck pain score (NRS score of 7.9 [D group] vs 6.6 [ND group], $p=0.015$; Fig. 5). Statistical difference between cohorts faded at 3 months following surgery (5.0 [D group] vs 4.3 [ND group], $\mathrm{p}=0.331$ ), and throughout 6 -month $(\mathrm{p}=0.853)$ and 1-year $(\mathrm{p}=0.326)$ time points. 


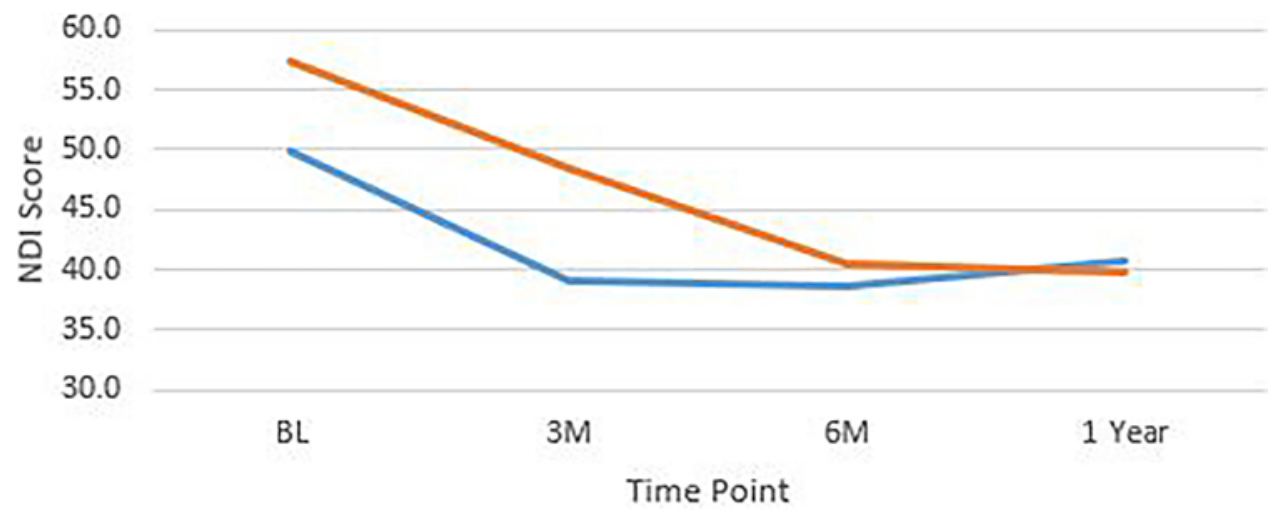

Not Depressed Depressed

FIG. 2. Comparison of improvement of NDI scores in both groups after cervical deformity correction, from baseline (BL) to 1 year after surgery.

\section{Case Example}

Figure 6 compares 2 cases of cervical deformity in patients with (Patient A) and without (Patient B) baseline depression. Patient A was a 54-year-old man with baseline depression. Patient B was a 50-year-old man without baseline depression. Both patients underwent a posterior C2-T2 fusion. Patient A's baseline HRQOL scores were as follows: NDI score of 62, mJOA score of 13, EQ-5D score of 0.546 , and NRS neck pain score of 9. Patient B's baseline HRQOL scores were as follows: NDI score of 54, mJOA score of 11, EQ-5D score of 0.659, and neck pain score of 6. Patient A had a worse baseline EQ-5D score, but all other metrics were similar to those of Patient B. At 1 year, Patients A and B had achieved similar outcomes: NDI scores of 36 and 28, EQ-5D scores of 0.803 and 0.820 , mJOA scores of 14 and 16, and neck pain scores of 3 and 3 , respectively.

\section{Discussion}

The psychological burden of spinal deformity has been well established in the lumbar spine, where pathologies of depression, anxiety, or fear-of-movement correlate to decreased postoperative improvement and increased preoperative pain and disability levels..$^{10,20,26,32}$ Similar effects are speculated to exist in similar populations undergoing cervical deformity surgery but remain less defined. Our study followed the recovery of patients with or without history of anxiety/depression undergoing cervical deformity surgery. At treatment onset, the depressed cohort reported lower mean EQ-5D scores and higher levels of pain, despite having similar levels of malalignment and myelopathy. During recovery of 6-12 months following surgery, these differences diminished to the point that there were no longer observable differences between the cohorts.

Our baseline data demonstrate that patients with depression and anxiety express significantly higher levels of neck pain and lower overall QOL on the EQ-5D assessment, despite having similar malalignments and myelopathies. Similarly, past findings have shown association between high occurrences of intense pain and low QOL scores in individuals who have sustained a spinal cord injury, ${ }^{4,24,27}$ as well as those who present with neck pain ${ }^{8,19}$ and cervical myelopathy. ${ }^{30}$

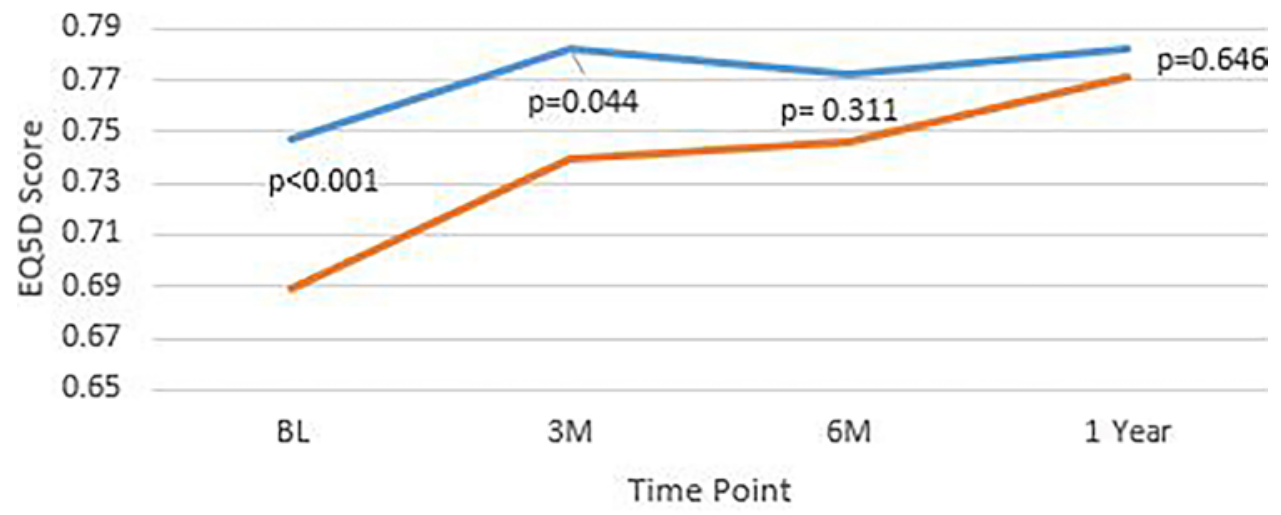

Not Depressed Depressed

FIG. 3. Comparison of improvement of EQ-5D scores in both groups after cervical deformity correction, from baseline to 1 year after surgery. 


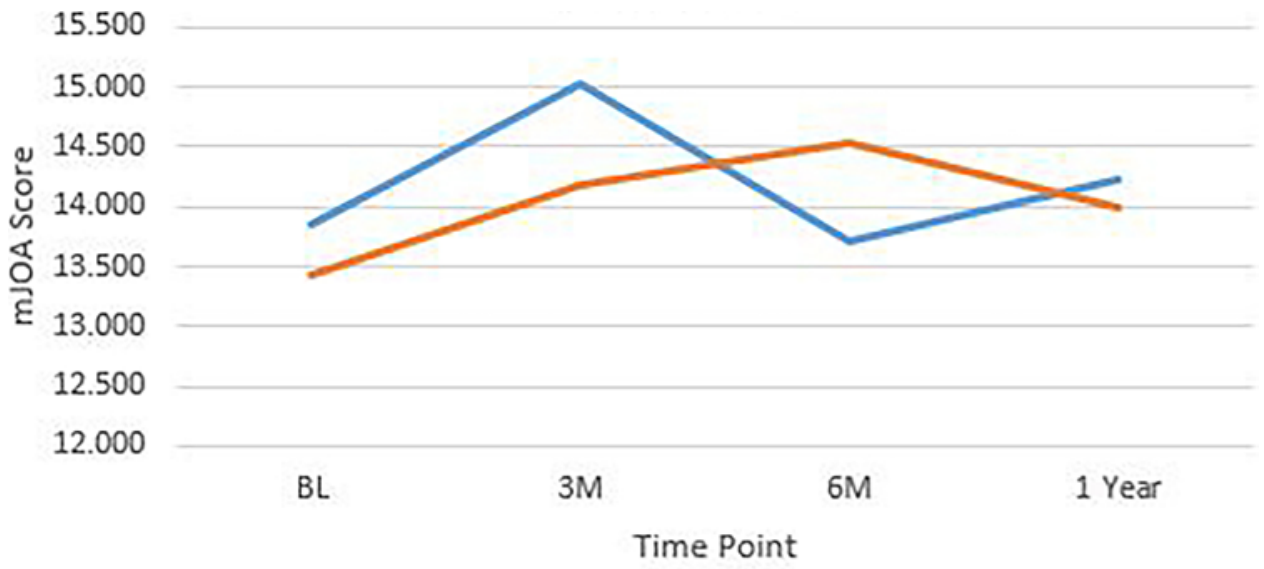

\section{Not Depressed Depressed \\ FIG. 4. Comparison of improvement of mJOA scores in both groups after cervical deformity correction, from baseline to 1 year after surgery.}

These data do not imply that patients with psychological distress have similar postoperative recovery pathways to those without such issues. To better manage the potential adverse impacts of psychological distress and pain experience in individuals following cervical deformity surgery, preoperative psychological assessment is advocated. Once identified, these psychological distress factors can be mitigated with cognitive behavioral therapy prior to surgery. Implementation of cognitive behavioral therapy could result in a lower level of pain experienced by depressed individuals suffering from cervical deformity, as has already been seen in patients who have undergone lumbar discectomy ${ }^{5}$ and cervical spine surgery. ${ }^{1}$

The fear-avoidance model expands on how fear and anxiety related to experiencing potential pain can cause a person to reduce the use of an affected area, resulting in the deconditioning of muscle and an increase in pain. Lack of movement can worsen a patient's condition and, by extension, inhibit recovery. This behavior can also inhibit an individual from taking part in positive experiences, or positive reinforcers, leading to negative mood changes and ultimately depression. ${ }^{9}$ Depression, being associated with an elevated experience of pain, ${ }^{6,15}$ can then feed into the fear-avoidance mechanism, further inhibiting patient improvement. This framework identifies fear, anxiety, and depression as obstacles affecting any individual experiencing consistent pain.

As data collection continued, at 3 months postsurgery we found NDI scores, mJOA scores, and pain levels to be similar in both groups, whereas mean EQ-5D scores remained significantly lower in the cohort with depression. Since the EQ-5D assesses an individual's QOL by taking into account measurements for anxiety and depression, this result was expected. Similarly, the recent literature has reported low mean EQ-5D scores in populations with high depression rates. Specifically, Jansson et al. recorded a low mean EQ-5D score (0.36) in a cohort of patients with lumbar spinal stenosis in which over half of the individuals were reported to be depressed. ${ }^{16}$ Any observable difference between groups on EQ-5D scores had diminished by the 6-month mark. Contrary to the outcomes found in the surgical correction of lumbar spine deformity, as well as the findings of Alvin et al. concluding that more severe preoperative depression was associated with worse surgical

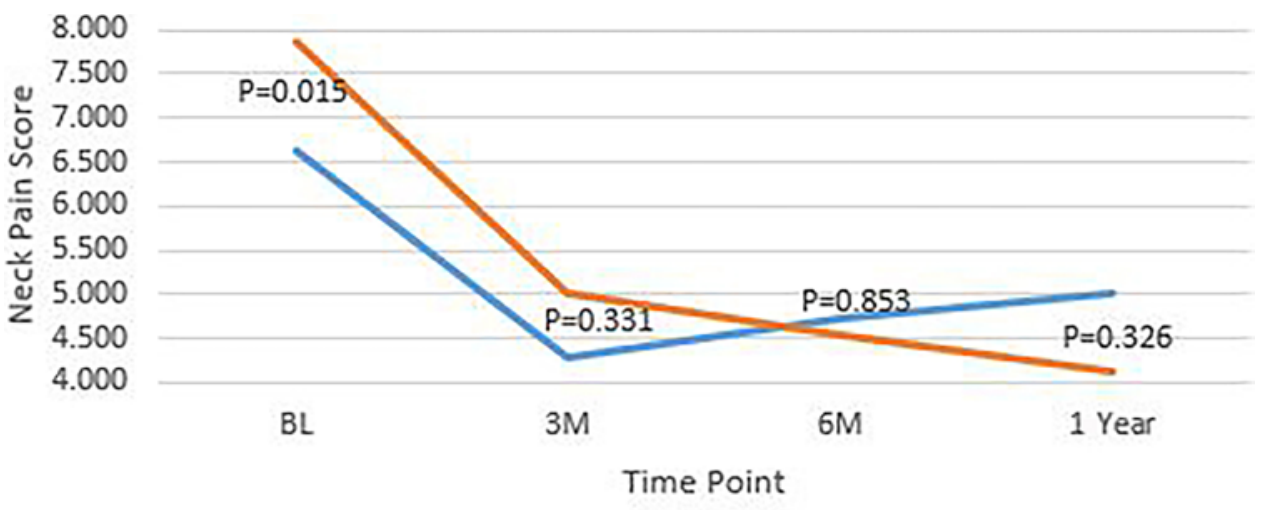

Not Depressed $\longrightarrow$ Depressed

FIG. 5. Comparison of improvement in NRS neck pain scores in both groups after cervical deformity correction, from baseline to 1 year after surgery. 

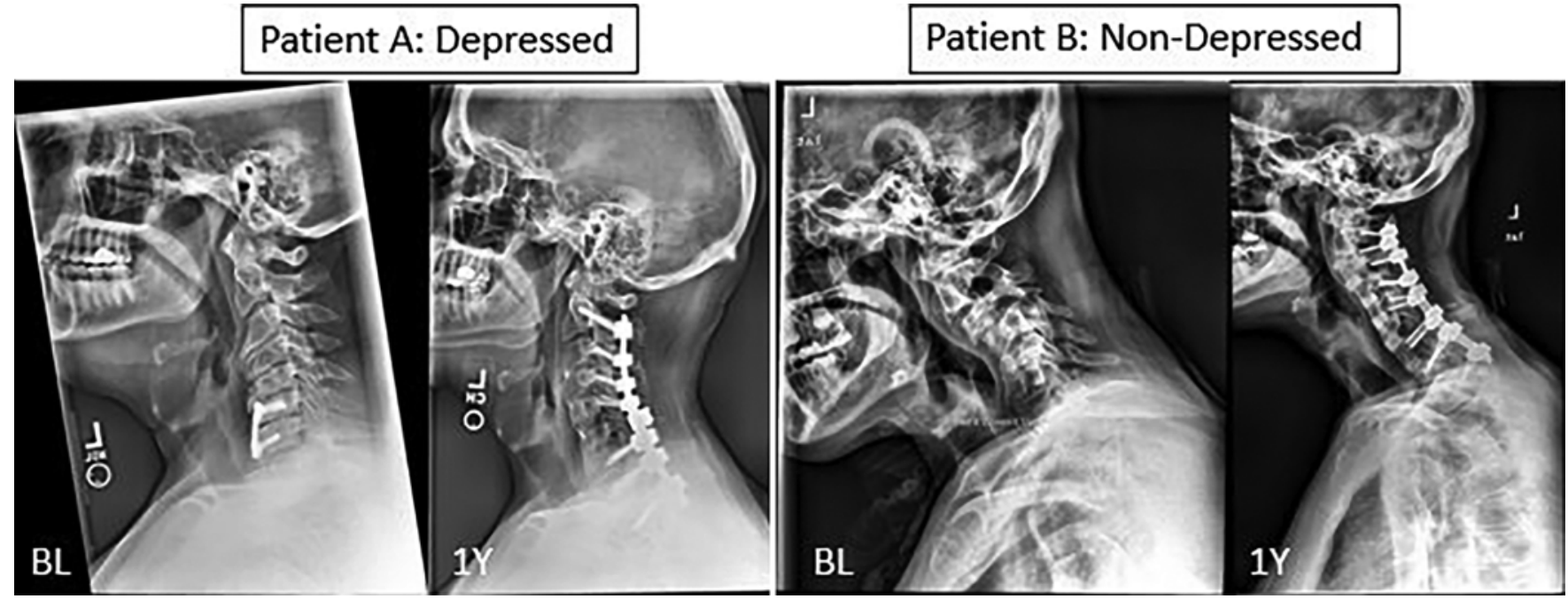

FIG. 6. Examples of 2 cases involving cervical deformity: one in a patient with baseline depression and another in a patient without depression.

outcomes 1 year following posterior cervical fusion, ${ }^{2}$ the $\mathrm{D}$ and ND groups were found to be statistically similar in all levels of pain and improvement 1 year postoperatively.

These results indicate that having depression as a comorbidity did not alter the short-term outcome in patients undergoing cervical deformity correction. McGregor and Hughes reported a similar finding and concluded that psychological factors could not be used to predict the surgical outcomes of nerve root compression interventions, negative or otherwise. ${ }^{23}$ This finding, in conjunction with the data obtained from our study, opposes our initial hypothesis. More appropriately, our results indicate that cervical deformity is a major underlying cause in the depression of individuals with comorbidities. Variance among the pain experiences of depressed and nondepressed individuals has already been established in the literature, ${ }^{6,15}$ as well as having been observed in the present study. Conveniently, this difference is resolved by 3 months following surgery. Thus, it appears that surgical correction of cervical deformity alleviated symptoms of depression in affected individuals. The statistically similar results observed in both $\mathrm{D}$ and ND groups on the final EQ-5D evaluations support this assertion. Past research is consistent with this outcome, indicating that patients undergoing various kinds of back surgery report improvements in levels of anxiety and depression 1 year following the procedure. ${ }^{17,25}$ These findings, in correlation with the association of poor mental health and musculoskeletal pain, ${ }^{7}$ all provide strong evidence that cervical deformity is $\mathrm{q}$ an underlying cause in the depression of its patients. To further understand this model of depression, future research is needed.

\section{Limitations}

Solidifying the etiology of depression in individuals with cervical deformity requires data regarding the initial onset of depression in relation to symptoms of pain. Psychiatric evaluation by a trained professional prior to surgery would also be helpful in identifying the specific depressive disorder, along with its severity, present in each individual, resulting in a more specific data analysis. Professional psychiatric opinion would also help to eliminate the inherent bias of self-reported data potentially present in our EQ-5D surveys.

Another potential limitation is that the depression group contained individuals with both current and/or past depression. Because patients with past depression may no longer be depressed, they may provide conflicting responses on the HRQOL assessment questionnaires. These individuals could either be eliminated from study or comprise a third cohort. This study is also limited by a lack of knowledge of medications individuals might be taking for their depression. Patients who have received treatment could yield results more similar to those derived from our ND group, potentially minimizing any observed differences. To comprehensively prove a long-lasting elimination of depression in these individuals, data on their future psychological status in relation to their state of recovery are necessary.

\section{Conclusions}

The data from the present study indicate that individuals with a history of depression experience higher levels of pain and a lower HRQOL than their nondepressed counterparts despite sharing a similar condition. Any difference in HRQOL parameters and pain experience between depressed and nondepressed cohorts was reversed 6 months following surgery. These findings strongly suggest that cervical deformity correction alleviated some symptoms of depression in the D group. Consequently, cervical deformity can be considered a major underlying cause in the depression of comorbid individuals. More research is needed to further solidify this relationship.

\section{References}

1. Adogwa O, Elsamadicy AA, Cheng J, Bagley C: Pretreatment of anxiety before cervical spine surgery improves clinical outcomes: a prospective, single-institution experience. World Neurosurg 88:625-630, 2016 
2. Alvin MD, Miller JA, Sundar S, Lockwood M, Lubelski D, Nowacki AS, et al: The impact of preoperative depression on quality of life outcomes after posterior cervical fusion. Spine J 15:79-85, 2015

3. Ames CP, Smith JS, Eastlack R, Blaskiewicz DJ, Shaffrey CI, Schwab F, et al: Reliability assessment of a novel cervical spine deformity classification system. J Neurosurg Spine 23:673-683, 2015

4. Anke AG, Stenehjem AE, Stanghelle JK: Pain and life quality within 2 years of spinal cord injury. Paraplegia 33:555559,1995

5. Archer KR, Motzny N, Abraham CM, Yaffe D, Seebach CL, Devin CJ, et al: Cognitive-behavioral-based physical therapy to improve surgical spine outcomes: a case series. Phys Ther 93:1130-1139, 2013

6. Bair MJ, Robinson RL, Katon W, Kroenke K: Depression and pain comorbidity: a literature review. Arch Intern Med 163:2433-2445, 2003

7. Björnsdóttir SV, Jónsson SH, Valdimarsdóttir UA: Mental health indicators and quality of life among individuals with musculoskeletal chronic pain: a nationwide study in Iceland. Scand J Rheumatol 43:419-423, 2014

8. Blozik E, Laptinskaya D, Herrmann-Lingen C, Schaefer H, Kochen MM, Himmel W, et al: Depression and anxiety as major determinants of neck pain: a cross-sectional study in general practice. BMC Musculoskelet Disord 10:13, 2009

9. Boersma K, Linton SJ: Psychological processes underlying the development of a chronic pain problem: a prospective study of the relationship between profiles of psychological variables in the fear-avoidance model and disability. Clin J Pain 22:160-166, 2006

10. Burton AK, Tillotson KM, Main CJ, Hollis S: Psychosocial predictors of outcome in acute and subchronic low back trouble. Spine (Phila Pa 1976) 20:722-728, 1995

11. Chapman CR, Cox GB: Anxiety, pain, and depression surrounding elective surgery: a multivariate comparison of abdominal surgery patients with kidney donors and recipients. J Psychosom 21:7-15, 1977

12. Childs JD, Cleland JA, Elliott JM, Teyhen DS, Wainner RS, Whitman JM, et al: Neck pain: clinical practice guidelines linked to the International Classification of Functioning, Disability, and Health from the Orthopedic Section of the American Physical Therapy Association. J Orthop Sports Phys Ther 38:A1-A34, 2008

13. Elsamadicy AA, Adogwa O, Cheng J, Bagley C: Pretreatment of depression before cervical spine surgery improves patients' perception of postoperative health status: a retrospective, single institutional experience. World Neurosurg 87:214-219, 2016

14. Furlan JC, Kalsi-Ryan S, Kailaya-Vasan A, Massicotte EM, Fehlings MG: Functional and clinical outcomes following surgical treatment in patients with cervical spondylotic myelopathy: a prospective study of 81 cases. J Neurosurg Spine 14:348-355, 2011

15. Haythornthwaite JA, Sieber WJ, Kerns RD: Depression and the chronic pain experience. Pain 46:177-184, 1991

16. Jansson KA, Németh G, Granath F, Jönsson B, Blomqvist P: Health-related quality of life (EQ-5D) before and one year after surgery for lumbar spinal stenosis. J Bone Joint Surg Br 91:210-216, 2009

17. Jansson KA, Németh G, Granath F, Jönsson B, Blomqvist $P$ : Health-related quality of life in patients before and after surgery for a herniated lumbar disc. J Bone Joint Surg Br 87:959-964, 2005

18. Kumar VG, Rea GL, Mervis LJ, McGregor JM: Cervical spondylotic myelopathy: functional and radiographic longterm outcome after laminectomy and posterior fusion. Neurosurgery 44:771-778, 1999

19. Linton SJ: A review of psychological risk factors in back and neck pain. Spine (Phila Pa 1976) 25:1148-1156, 2000
20. Linton SJ, Shaw WS: Impact of psychological factors in the experience of pain. Phys Ther 91:700-711, 2011

21. MacGregor AJ, Andrew T, Sambrook PN, Spector TD: Structural, psychological, and genetic influences on low back and neck pain: a study of adult female twins. Arthritis Rheum 51:160-167, 2004

22. Matsumoto M, Okada E, Ichihara D, Watanabe K, Chiba K, Toyama Y, et al: Age-related changes of thoracic and cervical intervertebral discs in asymptomatic subjects. Spine (Phila Pa 1976) 35:1359-1364, 2010

23. McGregor AH, Hughes SPF: The evaluation of the surgical management of nerve root compression in patients with low back pain: part 2: patient expectations and satisfaction. Spine (Phila Pa 1976) 27:1471-1477, 2002

24. Middleton J, Tran Y, Craig A: Relationship between quality of life and self-efficacy in persons with spinal cord injuries. Arch Phys Med Rehabil 88:1643-1648, 2007

25. Nork SE, Hu SS, Workman KL, Glazer PA, Bradford DS: Patient outcomes after decompression and instrumented posterior spinal fusion for degenerative spondylolisthesis. Spine (Phila Pa 1976) 24:561-569, 1999

26. Pincus T, Burton AK, Vogel S, Field AP: A systematic review of psychological factors as predictors of chronicity/disability in prospective cohorts of low back pain. Spine (Phila Pa 1976) 27:E109-E120, 2002

27. Rintala DH, Loubser PG, Castro J, Hart KA, Fuhrer MJ: Chronic pain in a community-based sample of men with spinal cord injury: prevalence, severity, and relationship with impairment, disability, handicap, and subjective well-being. Arch Phys Med Rehabil 79:604-614, 1998

28. Sether LA, Yu S, Haughton VM, Fischer ME: Intervertebral disk: normal age-related changes in MR signal intensity. Radiology 177:385-388, 1990

29. Siivola SM, Levoska S, Tervonen O, Ilkko E, Vanharanta H, Keinänen-Kiukaanniemi S: MRI changes of cervical spine in asymptomatic and symptomatic young adults. Eur Spine J 11:358-363, 2002

30. Stoffman MR, Roberts MS, King JT Jr: Cervical spondylotic myelopathy, depression, and anxiety: a cohort analysis of 89 patients. Neurosurgery 57:307-313, 2005

31. Trief PM, Grant W, Fredrickson B: A prospective study of psychological predictors of lumbar surgery outcome. Spine (Phila Pa 1976) 25:2616-2621, 2000

32. Vlaeyen JWS, Linton SJ: Fear-avoidance model of chronic musculoskeletal pain: 12 years on. Pain 153:1144-1147, 2012

\section{Disclosures}

The ISSG is funded through research grants from DePuy Synthes. Dr. Ames is an employee of UCSF; is a consultant for Stryker, Medtronic, and DePuy; is a patent holder with Fish \& Richardson; and has received royalties from Stryker and Biomet Spine. Dr. Daniels is a consultant for Spineart, Stryker, and Orthofix; he has received support from Orthofix for clinical or research efforts unrelated to the current study. Dr. Eastlack has an ownership stake in Spine Innovation; is a consultant for NuVasive, Alphatec, Aesculap, K2M, Titan, and Seaspine; owns stock in NuVasive, Alphatec, and Seaspine; and is a patent holder with Globus, Spine Innovation, NuTech, and Invuity. Dr. Kim is a consultant for K2M and Zimmer-Biomet; he has received support from CSRS for clinical or research efforts unrelated to the current study, and is on the boards of HSS Journal, Asian Spine Journal, and Global Spine Journal. Dr. Mundis is a consultant for NuVasive, K2M, and Allosource. Dr. Neuman has received support from DePuy Synthes for clinical or research efforts unrelated to the current study. Dr. Passias is a consultant for Medicrea and SpineWave; has received support for educational course work; and has received support from CSRS for clinical or research efforts unrelated 
to the current study. Dr. Sciubba is a consultant for the following companies: Medtronic, DePuy Synthes, Stryker, NuVasive, Globus, and K2M. Dr. Shaffrey is a consultant for Medtronic, NuVasive, and K2M; owns stock in NuVasive; and is a patent holder with NuVasive, Medtronic, and Zimmer-Biomet. Dr. Smith is a consultant for Zimmer-Biomet, NuVasive, and K2M; receives royalties from Zimmer-Biomet; has has received fellowship funding from NREF and from AOSpine; has received research support for the current study from DePuy Synthes/ISSG; and has received support from DePuy Synthes/ISSG for clinical or research efforts unrelated to the current study.

\section{Author Contributions}

Conception and design: Passias, Poorman, Horn, Daniels, Hamilton, Kim, Sciubba, Bortz, Segreto, Kelly, Smith, Neuman, Shaffrey, V Lafage, R Lafage, Ames, Hart, Mundis, Eastlack. Acquisition of data: Passias, Poorman, Horn, Daniels, Hamilton, Kim, Sciubba, Diebo, Bortz, Segreto, Kelly, Smith, Neuman, Shaffrey,
V Lafage, R Lafage, Ames, Hart, Eastlack. Analysis and interpretation of data: Passias, Poorman, Horn, Frangella, Diebo. Drafting the article: Passias, Poorman, Horn, Frangella, Diebo, Neuman. Critically revising the article: Passias, Poorman, Horn, Frangella, Hamilton, Kim, Sciubba, Diebo, Bortz, Segreto, Kelly, Smith, Neuman, V Lafage, R Lafage, Ames, Mundis, Eastlack. Reviewed submitted version of manuscript: all authors. Approved the final version of the manuscript on behalf of all authors: Passias. Statistical analysis: Passias, Poorman, Horn, R Lafage. Administrative/ technical/material support: Passias, Daniels, Smith, Shaffrey, V Lafage, R Lafage, Ames, Mundis. Study supervision: Passias, Smith, V Lafage, Ames, Hart.

\section{Correspondence}

Peter G. Passias, New York Spine Institute, NYU Medical CenterHospital for Joint Diseases, Department of Orthopaedic Surgery, 301 East 17th St., New York, NY 10003.email: pgpassias@ yahoo.com. 